\title{
Applying Image Fusion Technique with MRFKMC for Change Detection in SAR Images
}

\author{
Vasireddy Pravalya \\ M.Tech Student \\ Vardhaman College of \\ Engineering \\ Hyderabad, India
}

\author{
J Krishna Chaithanya \\ Associate Professor \\ Vardhaman College of \\ Engineering \\ Hyderabad, India
}

\author{
T. Ramashri, PhD \\ Professor \\ Sri Venkateshwara University \\ Tirupathi, India
}

\begin{abstract}
In this paper, a novel framework for change detection in synthetic aperture radar (SAR) images based on image fusion and clustering algorithms have been carried out. The significance of image fusion technique is to generate a difference image (DI) by using complementary information from a mean-ratio image and a log-ratio image. Dual - tree complex discrete wavelet transform (DTCWT) fusion technique is considered in this paper. To restrain the background information and enhance the information of changed regions in the fused image, DTCWT fusion algorithm is applied on ratio images. The approach then classifies changed and unchanged regions by Markov random field K-means (MRFKMC) clustering algorithm. Theoretical analysis experiments are carried out on SAR images by applying MRFKMC and compared the results with MRFFCM.
\end{abstract}

\section{Keywords}

Dual tree complex wavelet transform, difference image, image fusion, K-means clustering, Markov random field, synthetic aperture radar.

\section{INTRODUCTION}

Image change detection means detecting the changes in images of the same scene that are taken at different times. This is of widespread interest due to a large number of applications in diverse disciplines, such as remote sensing, medical diagnosis [9] and video surveillance [10]. Generally, change detection in SAR images is the process of the analysis of two coregistered SAR images which are obtained over the same geographical area at different times. Such analysis is unsupervised when it aims to discriminate between two opposite classes (which represent unchanged and changed areas) with no prior knowledge about the scene.

For the remote sensing images, differencing (subtraction operator) and rationing (ratio operator) are well-known techniques for producing a difference image. In differencing, changes are measured by subtracting the intensity values pixel by pixel between the considered images. In rationing, changes are obtained by applying a pixel-by-pixel ratio operator on the temporal images. However, in the case of SAR images, the ratio operator is typically used instead of the subtraction operator since the image differencing technique is not adapted to the statistics of SAR images and nonrobust to calibration errors.
As is mentioned in [2], the procedure of change detection in SAR images can be divided into three steps: 1) image preprocessing; 2) generation of a difference image (DI) from multitemporal images; and 3) analysis of the DI. The images which are considered are first preprocessed and later from that difference image i.e., changed area is generated. In the last step that DI is analyzed by applying algorithms to the changed image.

The DI-analysis step can be looked on as the process of image segmentation. We have got two conventional methods for that, the threshold method and the clustering method. In the threshold method, some essential models are usually established to search for a best threshold to divide DI into two classes. And in the clustering method, we don't need to establish a model, so it is more convenient and feasible. One of the most popular clustering methods for image segmentation is the fuzzy c-means (FCM) algorithm, which can retain more image information than hard clustering in some cases. MRF provides a basis for modeling information about the mutual influences among image pixels. An important issue of MRF is the energy function which directly characterizes the way to utilize spatial context. Considering the severe speckle noise in SAR images, determining the relationship among pixels is a complex process. Such complexity appears as two aspects: firstly, in the homogeneous region in DI, outliers disturb the utilization of the energy function, and it is not easy to stem such corruption; secondly, in the heterogeneous region in DI, an obscure boundary will emerge between two classes instead of an exact one [1]. So in order to reduce the effect of speckle noise, Markov Random Field FCM algorithm [1] is used here. This approach does not improve FCM by modifying the objective function. Instead, it focuses on the modification of the membership to reduce the effect of speckle noise. It computationally simple, its objective function can just return to the original form of FCM which leads to its less time consumption than some other improved FCM algorithms. It modifies the membership of each pixel by introducing the information provided by the spatial context, i.e., the neighbors of the central pixel as well as their interrelationship are concerned in the process of using MRF.

\section{LITERATURE SURVEY}

The performance of the proposed system mainly depends on the quality of difference image (DI) \& accuracy of the classification method. Two conventional methods for difference image analysis are 1) Threshold method, 2) Clustering method. In the threshold method [3], some 
essential models are usually established to search for a best threshold to divide DI into two classes. E.g.:-minimum error thresholding algorithm (K\&I), expectation maximization (EM) algorithm. Advantages of this approach are that it is simple and effective tool to separate objects from the background. But this approach lack objective measures to assess the performance. Noise, ambient illumination, busyness of gray levels within the object and its background, inadequate contrast etc complicate the thresholding operation. Also improper thresholding causes blotches, streaks etc on the resulting image.

But in the clustering method, we don't need to establish a model, so it seems to be more convenient and feasible. One of the most popular clustering methods for image segmentation is the fuzzy c-means (FCM) algorithm [4], which can retain more image information than hard clustering in some cases.

Therefore, enlightened by [5], [6], and [11], in order to reduce the effect of speckle noise, we propose a novel form of the energy function of the MRF to modify the membership of the FCM algorithm instead of modifying the objective function.

In [7], Cai et al. proposed the fast generalized FCM algorithm (FGFCM) for image segmentation which incorporates the spatial information, the intensity of local pixel neighborhood, and the number of gray levels in an image.

In [8], Krindis and Chatzis proposed a robust fuzzy local information C-means clustering algorithm (FLICM) for image segmentation. The characteristic of FLICM is the use of a fuzzy local similarity measure which is aimed to guarantee noise insensitiveness and image detail preservation. The reformulated FLICM (RFLICM) [16] incorporates the information about spatial context by adding a new fuzzy factor into its objective function for the purpose of enhancing the changed information and reducing the effect of speckle noise.

A MRF serves as an opportune tool to introduce information about the mutual influences among image pixels in a powerful and formal way. In [5], Chatzis and Varvarigou proposed a novel fuzzy objective function regularized by KullbackLeibler divergence information. Their algorithm was facilitated by the application of a mean-field-like approximation of the MRF prior. In [6], Markov spatial constraint field and the fuzzy segmentation information resulting from FCM are fused. In [11], FCM with the MRF was applied in wavelet domain for image segmentation. Its label field of image was characterized by the MRF. The modified objective function with locally spatial constraint was introduced by the initial label of different scale wavelet coefficients.

\section{PROPOSED METHODOLOGY}

Let us consider two images $X_{1}$ and $X_{2}$, where $X_{1}$ denotes the original SAR image 1 and is given by $X_{1}=\left\{X_{1}(i, j), 1<i<\right.$ $h, 1<j<w\}$ and $X_{2}$ denotes original SAR image 2 and given by $\mathrm{X}_{2}=\left\{\mathrm{X}_{2}(\mathrm{i}, \mathrm{j}), 1<\mathrm{i}<\mathrm{h}, 1<\mathrm{j}<\mathrm{w}\right\}$ of size hxw which are acquired on two different dates with two time instants $t_{1}$ and $t_{2}$.

Figure1 shows the actual flow diagram of the change detection approach. It can be categorized into two phases, the first phase involves obtaining information from mean ratio operator and log ratio operator and applying fusion rule on them to generate a difference image, and second phase includes analyzing the final fused image by using an MRFKMC algorithm.

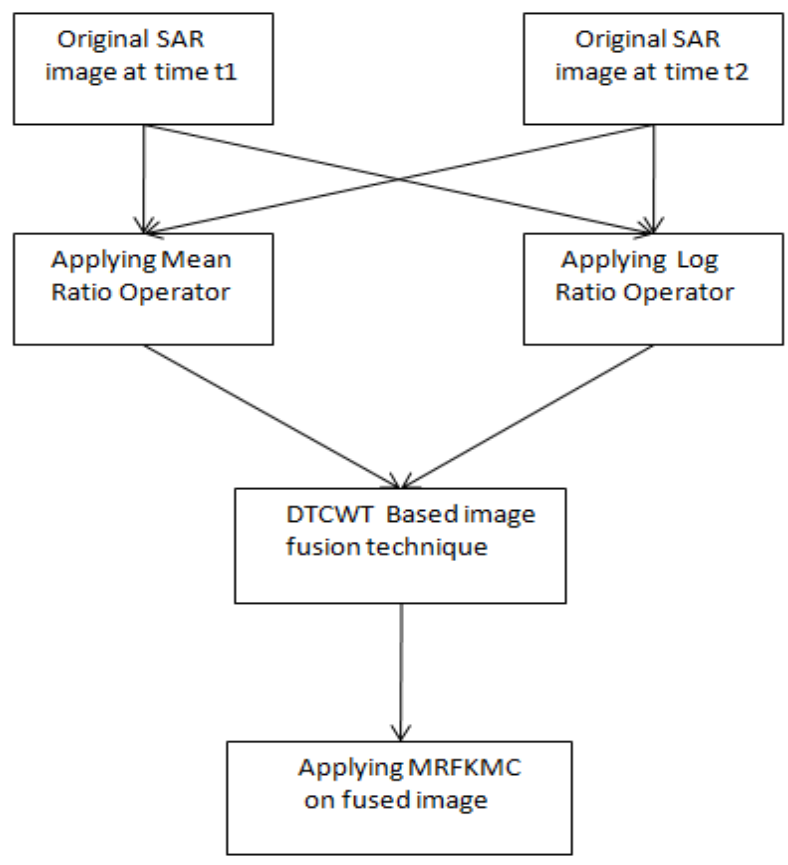

Figure 1. Flow Diagram of change detection approach

\subsection{Mean Ratio and Log Ratio Operators}

The mean-ratio operator should be applied to generate the mean ratio image. It can be defined as follows:

$$
\begin{aligned}
& X_{m} \\
= & 1 \\
- & \min \left(\frac{\mu_{1}(i, j)}{\mu_{2}(i, j)}\right)
\end{aligned}
$$

Where $\mu_{1}$ and $\mu_{2}$ represent the local mean values of the pixels in a neighborhood of point $(i, j)$ of multitemporal SAR images $\mathrm{X}_{1}$ and $\mathrm{X}_{2}$, respectively. Above equation shows that the mean ratio operator produces difference image by using the local mean information of each pair of neighboring pixels. The underlying idea of the optimal difference image is that unchanged pixels exhibit small values, whereas changed areas exhibit larger values. Mean-ratio shows changed region but it doesn't enhance it. Result is better than that of $\log$ ratio operator. Similarly the absolute valued log-ratio can be defined as:

$$
X_{l}=\left|\log \frac{X_{2}}{X_{1}}\right|=\left|\log X_{2}-\log X_{1}\right|
$$

The logarithmic operator is characterized by enhancing the low-intensity pixels while weakening the pixels in the areas of high intensity therefore, the information of changed regions that is obtained by the log-ratio image may not be able to reflect the real changed trends in the maximum extent because of the weakening in the areas of high intensity pixels.

\subsection{Image Fusion using DTCWT}

Image fusion is the process that combines information from multiple images of the same scene in order to extend the information content. It has been noted that, for some applications of the discrete wavelet transform, improvements 
can be obtained by using an expansive wavelet transform in place of a critically-sampled one. An expansive transform is one that converts an N-point signal into $\mathrm{M}$ coefficients with $\mathrm{M}$ $>\mathrm{N}$. There are several kinds of expansive DWTs; here we considered the dual-tree complex discrete wavelet transform.

The DTCWT of a signal $\mathrm{x}$ is implemented using two critically sampled DWTs in parallel on the same data. The transform is 2-times expansive because for an $\mathrm{N}$-point signal it gives $2 \mathrm{~N}$ DWT coefficients. If the filters in the upper and lower DWTs are the same, then no advantage is gained. However, if the filters are designed is a specific way, then the subband signals of the upper DWT can be interpreted as the real part of a complex wavelet transform, and subband signals of the lower DWT can be interpreted as the imaginary part. Equivalently, for specially designed sets of filters, the wavelet associated with the upper DWT can be an approximate Hilbert transform of the wavelet associated with the lower DWT. The dual-tree complex DWT can be used to implement 2D wavelet transforms where each wavelet is oriented, which is especially useful for image processing.

\subsection{Markov Random Field}

MRF describes a system by local interaction and is able to capture many features of the system of interest by simply adding appropriate terms representing spatial or contextual dependencies into it. For this work, we denote a set of sites $\mathrm{s}$ $=\{1, \ldots, n\}$ representing the primitive points and define the label assignment $x=\{x 1, \ldots, x n\}$ to all sites as a realization of a family of random variables defined on $s$. We also define the feature space that describes the sites and understand it as a random observation field with its realization $\mathrm{y}=\{\mathrm{y} 1 \ldots \mathrm{yn}\}$. We also denote a label set $\mathrm{L}=\{1 \ldots \mathrm{m}\}$, where each label corresponds to a class centroid. An optimal labeling should minimize the posterior energy $\mathrm{U}(\mathrm{x} \mid \mathrm{y})=\mathrm{U}(\mathrm{y} \mid \mathrm{x})+\mathrm{U}(\mathrm{x})$.

In general, an image $\mathrm{I}=\{\mathrm{I}(\mathrm{h}, 1), 1 \leq \mathrm{h} \leq \mathrm{A}, 1 \leq 1 \leq \mathrm{B}\}$ itself can be viewed as a field, and each pixel of image is an element. If and only if some property of each element is only related to the neighborhood ones and is of no relationship to the other ones in the field, we call the random field $p(x)$ an MRF. In this work, V (yi|xi) is the Euclidean distance between point and class centroid:

$$
\mathrm{V}(\mathrm{yi} \mid \mathrm{xi})=\|\mathrm{yi}-\mathrm{Cxi}\|=|\mathrm{yi}-\mathrm{Cxi}|
$$

The Hammersley-Clifford theorem has proved that a given random field is an MRF if and only if its (joint) probability distribution $p(x)$ is a Gibbs distribution, i.e., the joint distribution of an MRF is given by [15]

\subsection{MRFKMC}

\subsubsection{K-means clustering}

The normalized feature pointsets $\left(\mathrm{S}_{\text {norm }}\right.$ and $\left.\mathrm{M}_{\text {norm }}\right)$ are first concatenated together (concat). Redundant features are then removed using the "k-means" clustering techniques [13] on the fused pointset of an individual retaining only the centroid of the points from each cluster. These clusters are formed using spatial and orientation information of a point. The keypoint descriptor of each cluster's centroid is the average of keypoint descriptors of all the points in each cluster. The distance classifier used is Euclidean distance. The number of clusters is determined using the PBM cluster validity index [14].

By considering the SAR images change detection is observed by ratio-operators. Image fusion technique is applied on the
DI and later MRF function is calculated by neighborhood pixel and $\mathrm{K}$-means clustering is applied to calculate weight of each pixel and produce expected results. K-means clustering provides very accurate results than MRFFCM technique which was applied in previous paper.

\subsubsection{Neighborhood Elimination}

This technique is applied on the normalized pointset of image individually. That is, for each point of image , those point that lie within the neighborhood of a certain radius are removed giving $\mathrm{s}_{\text {norm }}$ 'and $\mathrm{m}_{\text {norm }}$ ', the reduced image pointsets. Spatial information is used to determine the neighbors of each considered point.

\section{EXPERIMENTAL STUDY}

In order to validate the effectiveness of the proposed synthetic aperture radar based change detection approach, the performance of the proposed method can be studied using numerical results on a data set. The analysis of change detection is done as follows. First, calculate the false negatives (FN) nothing but changed pixels that are undetected. Second, calculate false positives (FP) that is unchanged pixels wrongly classified as changed. To calculate percentage correct classification (PCC), true positives (TP) which are detected as the changed area in the reference image and result and true negatives (TN) gives the number of pixels that are detected as the unchanged area in both the reference image and the result are also needed. Overall error (OE) is calculated in order to observe the error value. Here, kappa statistic is used to measure the accuracy or agreement. If the change detection map and the reference image are in complete agreement, then the kappa value is 1 . If there is no agreement among change detection map and reference image, then the kappa value will be 0 .

$$
\begin{aligned}
& \mathrm{OE}=\mathrm{FP}+\mathrm{FN} \\
& \mathrm{PCC}=(\mathrm{TP}+\mathrm{TN}) / \mathrm{N} \\
& \mathrm{N}=\mathrm{TP}+\mathrm{TN}+\mathrm{FP}+\mathrm{FN} \\
& \mathrm{KC}=\mathrm{PCC}-\mathrm{PRE} / 1-\mathrm{PRE} \\
& \mathrm{PRE}=(\mathrm{TP}+\mathrm{FP}) \mathrm{Nc}+(\mathrm{FN}+\mathrm{TN}) \mathrm{Nu} / \mathrm{N}^{2}
\end{aligned}
$$

Here $\mathrm{N}_{\mathrm{c}}$ and $\mathrm{Nu}$ represents actual number of pixels belonging to changed and unchanged classes. Where $\mathrm{N}$ represents total number of pixels in an image.

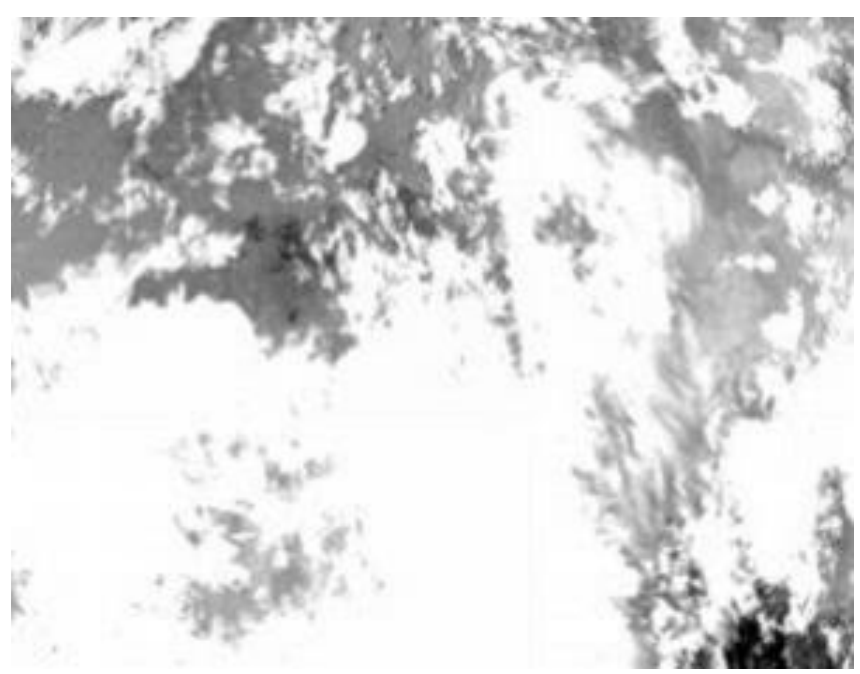

Figure 2. Original SAR Image1 


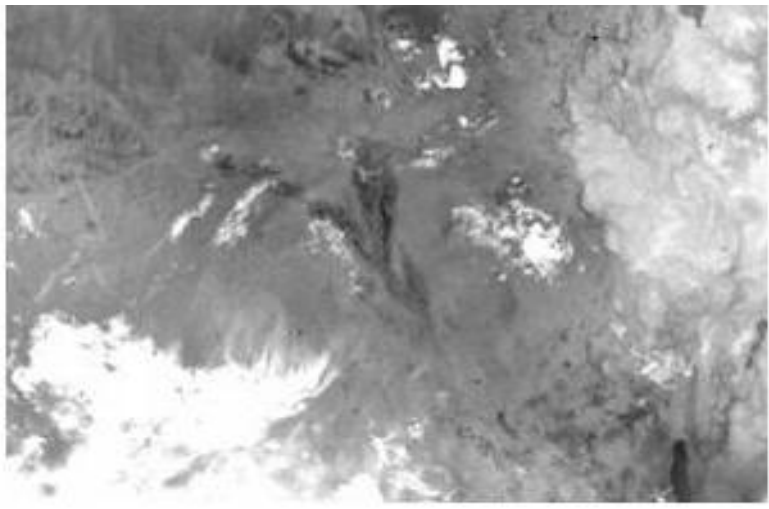

Figure 3. Original SAR Image2

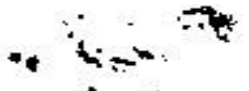

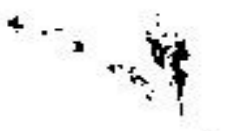

Figure 4. Log-ratio Image

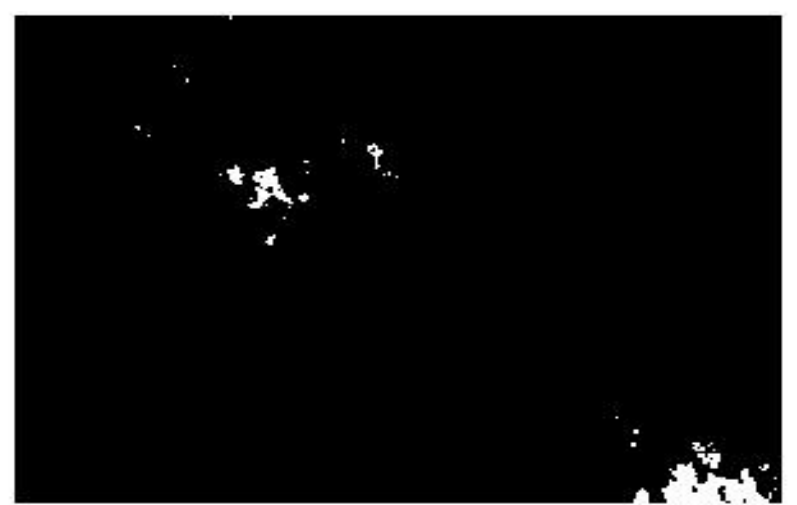

Figure 5. Mean Ratio Image

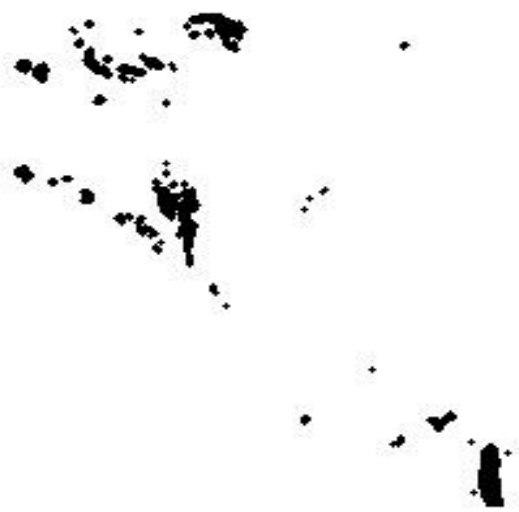

Figure 6. MRFFCM Image

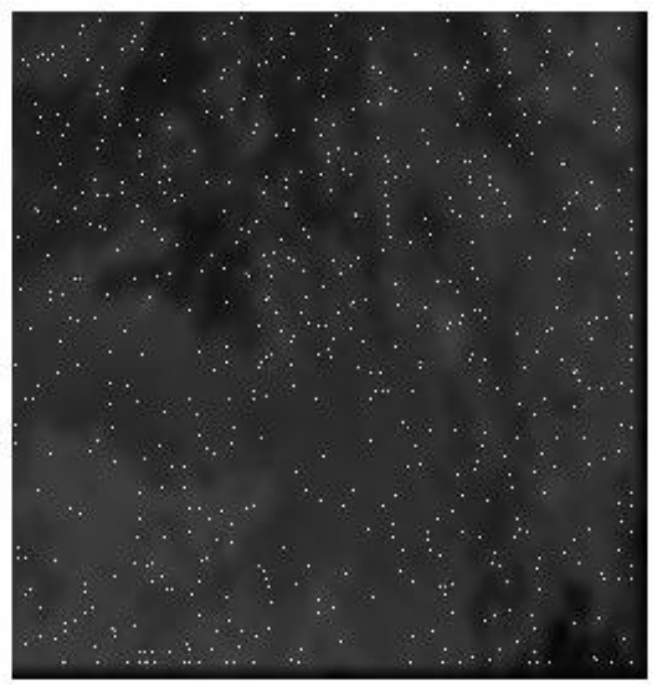

Figure 7. Fused Image

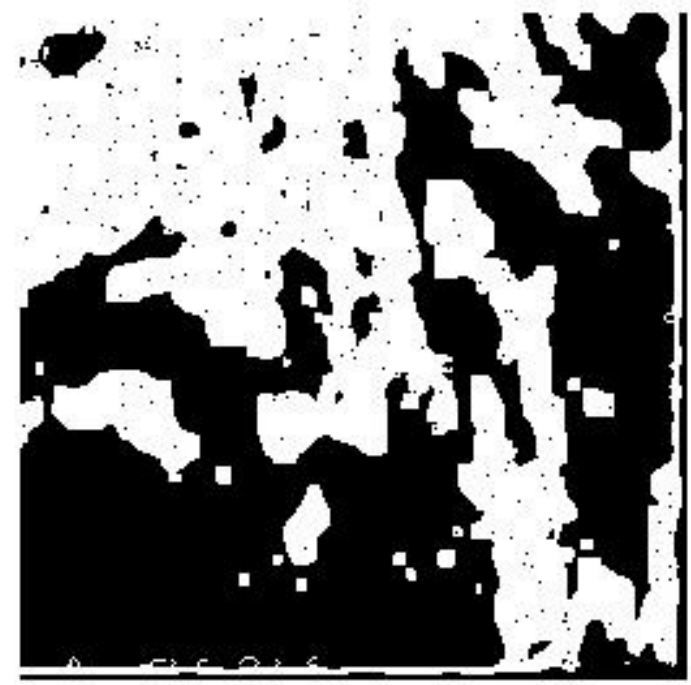

Figure 8. MRFKMC Image

Table 1: Change Detection Results of the Considered Image Set

\begin{tabular}{|c|c|c|c|c|c|c|}
\hline Algorithm & TP & FP & TN & FN & OE & PCC \\
\hline MRFFCM & 466.17 & 771.95 & 100 & 0.01 & 13057.8 & 0.776 \\
\hline MRFKMC & 989.27 & 0 & 22.8 & 0.53 & 107.29 & 0.995 \\
\hline
\end{tabular}

\section{CONCLUSION AND FUTURE SCOPE}

In this paper, multi temporal SAR images are mainly concentrated for change detection without any disturbance caused by the speckle noise. Mean ratio and $\log$ ratio operators are considered from whose information fusion technique takes the complementary information to proceed further in generating a difference image, in this fusion technique DTCWT is used which overcomes the 
disadvantages of other discrete wavelet transforms. K-means clustering provides accurate results than MRFFCM technique. This technique can also be applied to video, medical images.

\section{REFERENCES}

[1] Maoguo Gong; Linzhi Su; MengJia; Weisheng Chen,"Fuzzy Clustering With a Modified MRF Energy Function for Change Detection in Synthetic Aperture Radar Images," Fuzzy Systems, IEEE Transactions on vol.22, no.1, pp.98,109, Feb. 2014.

[2] L. Bruzzone and D. F. Prieto, "An adaptive semiparametric and contextbased approach to unsupervised change detection in multi-temporal remotesensing images," IEEE Trans. Image Process., vol. 11, no. 4, pp. 452-466, Apr. 2002.

[3] M. Sezgin and B. Sankur "A survey over image thresholding techniques and quantitative performance evaluation" 2004.

[4] J. C. Bezdek, Pattern Recognition with Fuzzy Objective Function. New York: Plenum, 1981.

[5] S. P. Chatzis and T. A. Varvarigou, "A fuzzy clustering approach toward hidden Markov random field models for enhanced spatially constrained image segmentation," IEEE Trans. Fuzzy Syst., vol. 16, no. 5, pp. 1351-1361, Oct. 2008.

[6] S. Liu, X. Li, and Z. Li, "A new image segmentation algorithm based the fusion of Markov random field and fuzzy c-means clustering," in Proc. IEEE Int. Symp. Commun. Inf. Technol., Oct. 2005, vol. 1, pp. 144-147.

[7] W. Cai, S. Chen, and D. Zhang, "Fast and robust fuzzy C-means clustering algorithms incorporating local information for image segmentation," Pattern Recog., vol. 40, no. 3, pp. 825-838, Mar. 2007.

[8] S. Krinidis and V. Chatzis, "A robust fuzzy local information C-means clustering algorithm," IEEE Trans.
Image Process., vol. 19, no. 5, pp. 1328-1337, May 2010 .

[9] D. Rey, G. Subsol, H.Delingette, andN.Ayache, "Automatic detection and segmentation of evolving processes in 3D medical images: Application to multiple sclerosis," Med. Image Anal., vol. 6, no. 2, pp. 163-179, Jun. 2002.

[10] D. M. Tsai and S. C. Lai, "Independent component analysis-based background subtraction for indoorsurveillance," IEEE Trans. Image Process., vol. 18, no. 1, pp. 158-167, Jan. 2009.

[11] X. Li and S. Bian, "Multiscale image segmentation using Markov random field and spatial fuzzy clustering in wavelet domain," in Proc. Int. Workshop Intell. Syst. Appl., May 2009, pp. 1-6.

[12] S. Geman and D. Geman, "Stochastic relaxation, Gibbs distributions and the Bayesian restoration of images," IEEE Trans. Pattern Anal. Mach. Intell., vol. PAMI-6, no. 6, pp. 721-741, Nov. 1984.

[13] A. Jain and R. Dubes, "Algorithms for clustering data", Prentice Hall, 1988.

[14] M. K. Pakhira, S. Bandyopadhyay and U. Maulik (2004). "Validity index for crisp and fuzzy clusters". Pattern Recognition, vol. 37, pp. 487-501.

[15] W. Cai, S. Chen, and D. Zhang, "Fast and robust fuzzy C-means clustering algorithms incorporating local information for image segmentation, Pattern Recog., vol. 40, no. 3, pp. 825-838, Mar. 2007.

[16] M. Gong, Z. Zhou, and J. Ma, "Change detection in synthetic aperture radar images based on image fusion and fuzzy clustering," IEEE Trans. Image Process., vol. 21, no. 4, pp. 2141-2151, Apr. 2012. 